\title{
Modeling and inversion 3D electromagnetic datasets on HPC platforms
}

\author{
Octavio Castillo-Reyes*, Daniel Blatter ${ }^{\dagger}$ \\ * Barcelona Supercomputing Center (BSC) \\ Nexus II Building c/Jordi Girona 29, 08034, Barcelona, Spain \\ e-mail: octavio.castillo@bsc.es \\ ${ }^{\dagger}$ Scripps Institution of Oceonography \\ University of California, San Diego, USA
}

\begin{abstract}
Modeling and inversion of 3D electromagnetic datasets on High-Performance Computing (HPC) have a fundamental role in solving the next generation of geoscience problems. These kind of problems are complex, multidisciplinary, and require collaboration to understand and solve the physical equations, pre-process and post-process the associated data with physical experiments, and build interpretations from the analysis of the numerical results.

In this talk, we present our advances in geophysical electromagnetic modelling and inversion using high-order vector finite elements (HEFEM), Markov chain Monte Carlo (MCMC), and HPC. The net result is an open-source parallel Python code, namely, PETGEM (Parallel Edge-based Tool for Geophysical Electromagnetic Modelling, http:// petgem.bsc.es). As the main arguments of this talk, we present: 1) the mathematical bases of our electromagnetic modeling and inversion algorithms; 2) real cases of 3D electromagnetic modeling and inversion on HPC architectures; 3) a study of the code's performance and accuracy on different HPC architectures. Finally, we provide summary remarks and conclusions.
\end{abstract}

\section{REFERENCES}

[1] Castillo-Reyes, O., Queralt, P., Marcuello, A., Ledo, J. (2020). Land CSEM simulations and experimental test using metallic casing in a geothermal exploration context: Vallès Basin (NE Spain) case study. Submitted to IEEE Transactions on Geoscience and Remote Sensing.

[2] Werthmüller, D., Rochlitz, R., Castillo-Reyes, O., \& Heagy, L. (2020). Open-source landscape for 3D CSEM modelling. arXiv preprint arXiv:2010.12926. Submitted to Geophysical Journal International.

[3] Castillo-Reyes, O., de la Puente, J., García-Castillo, L. E., Cela, J. M. (2019). Parallel 3D marine controlled-source electromagnetic modeling using high-order tetrahedral Nédélec elements. Geophysical Journal International, ggz285, vol 219: 39-65. ISSN 0956-540X.

[4] Castillo-Reyes, O., de la Puente, J., Cela, J. M. (2018). PETGEM: A parallel code for 3D CSEM forward modeling using edge finite elements. Computers \& Geosciences, vol 119: 123-136. ISSN 0098-3004. Elsevier. 